\title{
Nurses' perceptions of professional dignity in hospital settings
}

\section{Laura Sabatino}

Tor Vergata University, Italy

\author{
Mari Katariina Kangasniemi \\ University of Eastern Finland, Finland
}

\section{Gennaro Rocco}

Centre of Excellence for Nursing Scholarship, Italy

Rosaria Alvaro

Tor Vergata University, Italy

\author{
Alessandro Stievano \\ Centre of Excellence for Nursing Scholarship, Italy
}

\begin{abstract}
Background: The concept of dignity can be divided into two main attributes: absolute dignity that calls for recognition of an inner worth of persons and social dignity that can be changeable and can be lost as a result of different social factors and moral behaviours. In this light, the nursing profession has a professional dignity that is to be continually constructed and re-constructed and involves both main attributes of dignity.

Objectives: The purpose of this study was to determine how nurses described nursing's professional dignity in internal medicine and surgery departments in hospital settings.

Research design: The research design was qualitative.

Ethical considerations: This study was approved by the ethics committees of the healthcare organizations involved. All the participants were provided with information about the purpose and the nature of the study.

Participants: A total of 124 nurses participated in this study.

Method: The data were collected using 20 focus group sessions in different parts of Italy. The data were analysed by means of a conventional inductive content analysis starting from the information retrieved in order to extract meaning units and sorting the arising phenomena into conceptually meaningful categories and themes.

Results: Nursing's professional dignity was deeply embedded in the innermost part of individuals. Regarding the social part of dignity, a great importance was put on the values that compose nursing's professional identity, the socio-historical background and the evolution of nursing in the area considered. The social part of dignity was also linked to collaboration with physicians and with healthcare assistants who
\end{abstract}

Corresponding author: Laura Sabatino, Tor Vergata University, Via Montpellier I, Rome 00I72, Italy.

Email: laura.sabatino@fastwebnet.it 
were thought to have a central role in easing work strain. Equally important, though, was the relationship with peers and senior nurses.

Conclusion: The organizational environments under scrutiny with their low staffing levels, overload of work and hierarchical interactions did not promote respect for the dignity of nurses. To understand these professional values, it is pivotal to comprehend the role of different health professions in their cultural milieu and the evolution of the nursing profession in diverse countries.

\section{Keywords}

Absolute dignity, dignity of the human being, inter/intra-professional relationships, nursing, workplace elements

\section{Introduction}

The aim of this study was to describe and evaluate nurses' perceptions and experiences of professional dignity in the context of medical and surgical hospital environments. Italian researchers who were familiar with the environments under scrutiny formulated four questions to understand nurses' viewpoints of their own profession's dignity. The ultimate aim was to deepen understanding of professional dignity in nursing and thus to define and articulate the concept, especially in its social dimensions.

The concept of dignity is a multidimensional and intertwined construct. It has been debated and deemed controversial, with disagreements about its deep meanings. It has been described in different ways in theoretical articles, trying to explain this construct from different perspectives. ${ }^{1-5}$

Dignity has been described as the basis of human rights, intrinsic to the worth of human beings, but also associated with the social position a person occupies in society. ${ }^{6-8}$ Human dignity can be described as innate, inalienable ${ }^{9,10}$ and also as social and relative, and thus can be bestowed or achieved by merit or status. ${ }^{1-14}$ Absolute dignity calls for recognition of an inner worth of every person simply by virtue of being a person and can never be removed; dignity is 'incarnated' in persons. ${ }^{15}$

Social dignity, conversely, can be changeable and can be lost because of different social factors and moral behaviours. When seen in this light, dignity is defined as the capacity of behaving correctly and respectfully in relation to others and to make autonomous choices. Social dignity is considered as a value to be recognized by others, embedded in a time and a place and can be identified and expressed at various levels; it is conferred or gained by reciprocal actions in social contexts and is firmly based on relationships where each party enhances the other. ${ }^{11}$ Included in the concept of social dignity is the awareness of one's own personal dignity, implying recognition of one's own worth as a person. ${ }^{16-18}$

Dignity-of-self and dignity-in-relation ${ }^{11}$ are characteristics of the social dignity and are socially produced notions; both of them are contingent on the context ${ }^{11,19}$ of any situation and depend on cognition, emotions and behaviours. In this way, the social part of dignity is in contact with the vulnerability of human beings and, in our case, of nurses who at work are sometimes objectified, discriminated against and humiliated. ${ }^{20-22}$

When considering the concept of professional dignity in comparison with dignity in general, many values expressed as basic in all health professions are touched upon, such as altruism, accountability, excellence, duty, honour, integrity, respect for others, development of professional and social identity. ${ }^{23-25}$

Professional dignity and professional respect involve 'the quality of people, not only moral but also professional $[\ldots]$, with their excellence, their being better than others, that is to say the opposite of equality' (p. 19). ${ }^{17}$ The recognition of professional dignity starts by identifying the differences, from recognizing in the other a person who acts, and is an individual similar to me in his or her ability to act responsibly. ${ }^{17}$ 
From this point of view, nursing embeds a professional dignity that is to be continually constructed and re-constructed, reflected on, demonstrated and has to be updated regularly according to the dynamics of the society in which we are immersed. ${ }^{26}$ In Italy, nursing was first recognized as an autonomous profession in $1999,{ }^{27}$ followed by significant changes in professional practice. A single entry-level pathway for nurses via a 3-year university degree was instituted in 2001. In 2004, the first master's degrees in nursing science were realized and in 2006-2007, the first doctoral programmes were offered. Despite these advances, in many workplaces, there is still a large gap between the theory of nursing as autonomous and the practice of it. ${ }^{28}$

Emotional abuse of nurses is frequent, especially in acute care, where the professional dignity for nurses is often disregarded. ${ }^{29,30}$ Yet, it is precisely in these same settings that respect of nurses' professional dignity is pivotal in providing good quality patient care, good job satisfaction and good nurse retention within supportive organizations. ${ }^{31}$

For this reason, the research group aimed to elucidate the meaning of nursing's professional dignity in hospitals, and, in particular, in environments well-known for their rigid hierarchy and problematic interpersonal and informational communication, such as medical and surgical departments. ${ }^{32-36}$

\section{Method}

\section{Design}

A descriptive qualitative method was adopted as a research strategy. In this qualitative study, we used a conventional inductive approach to allow new insights to emerge about the phenomenon under scrutiny and focus groups (FGs) to collect information on the topic (Table 1).

\section{Sample}

The target group consisted of a purposive, convenience, non-probability sampling of clinical nurses working in medicine and general surgery departments and the data were collected in public facilities in 12 Italian regions. The regions were selected from the north, centre and south of Italy to obtain heterogeneity of data. In all, 15 public health organizations were involved and we had a total of 20 FGs (Table 2).

Altogether, 124 nurses, females and males, working in internal medicine and surgical wards took part in the FGs. A socio-demographic data sheet was used to record basic information.

\section{Inclusion and exclusion criteria}

Participants were selected in order to guarantee they had the best knowledge concerning the research argument. The criteria for participation were as follows: (a) clinical registered nurses and (b) working in internal medicine and general surgery departments for more than 1 year on a stable basis. Exclusion criteria were as follows: (a) to have managerial roles, (b) working in other clinical departments and (c) working in the same internal medicine and general surgery departments of two or more nurses in the same FG.

\section{Data collection}

Participants were recruited on a voluntary basis. The data were collected using FG discussions, in order to allow interactions among the informants. Every FG had between five and eight participants. The staff was informed of the topic by a leaflet distributed by the main researcher on the day of the session. A semistructured FG format, based on previous studies, ${ }^{37}$ was employed to prompt questions to stimulate group discussions. FGs were held in dedicated quiet rooms and were digitally recorded. At the end of each session, 


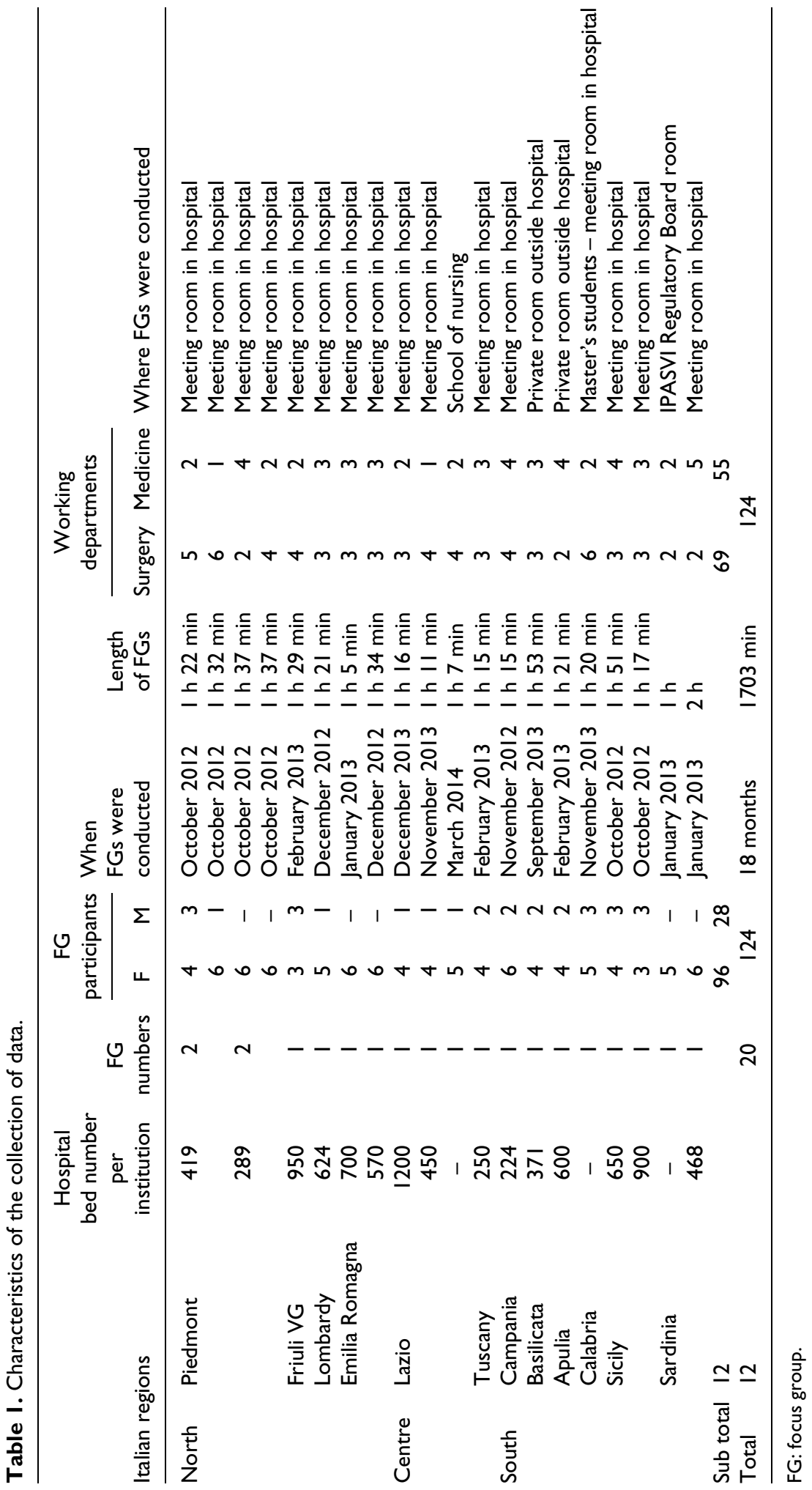


Table 2. Participants' characteristics $(n=124)$.

\begin{tabular}{|c|c|c|c|c|c|c|c|c|}
\hline \multirow{2}{*}{\multicolumn{2}{|c|}{ Nurses }} & \multicolumn{2}{|c|}{ Age } & \multicolumn{3}{|c|}{ Level of nursing education } & \multirow[b]{2}{*}{$\begin{array}{l}\text { Mean years } \\
\text { experience }\end{array}$} & \multirow[b]{2}{*}{$\begin{array}{c}\text { Mean working } \\
\text { experience (years) }\end{array}$} \\
\hline & & $\begin{array}{l}\text { Age range } \\
\text { (years) }\end{array}$ & $\begin{array}{c}\text { Mean age } \\
\text { (years) }\end{array}$ & $\begin{array}{l}\text { Nursing } \\
\text { diploma }\end{array}$ & $\begin{array}{l}\text { University } \\
\text { diploma }\end{array}$ & $\begin{array}{c}\text { Nursing } \\
\text { degree }\end{array}$ & & \\
\hline $\mathrm{F}$ & 96 & $26-56$ & 14 & 52 & 11 & 33 & 14 & $1-13 \quad 14-26 \quad 27-40$ \\
\hline$M$ & 28 & $24-60$ & 15 & 19 & I & 8 & 15 & \\
\hline Total & 124 & & & & & & & 54 \\
\hline
\end{tabular}

a summary of the meeting was given by the main researcher to obtain further elements to enrich the data collection.

\section{Data analysis}

It would have been possible to analyse the data using understandings gained from a previously used theoretical framework, ${ }^{18}$ but the researchers deemed that it was better to exclude prior views ${ }^{38}$ on the topic under scrutiny in order to gain new insights into the argument.

Using this methodological view, the data were processed by a conventional, pure, inductive content anal$\mathrm{ysis}^{39}$ starting from the information retrieved in order to extract meaning units, that is, "the constellations of words or statements that relate to the same central meaning ${ }^{40}$ (p. 106).

The data were transcribed and consisted of 12,429 lines and 413 A4 pages with 1.5 line spaces. A total of 1411 codes were reduced, connected together based on their similarities and differences, and abstracted to 49 sub-categories and 17 main categories. Seven connecting themes were found. The analytic process is shown graphically in Figure 1 to enable readers to comprehend result derivations.

Immediate verbatim transcriptions were read and reread by all Italian researchers several times in order to obtain a whole impression of the content. They were subsequently summarized in English for the fifth researcher. All investigators checked the contents, which enabled identification of unit analysis, sorting the arising phenomena into conceptually meaningful sub-categories, categories and themes. The categories reflected the perceptions about nursing's professional dignity and constituted the manifest content. The latent themes ${ }^{41}$ that emerged through abstraction were contextualized to the data and surmised on the basis of reflective reasoning. ${ }^{42}$

\section{Consideration of rigour}

To describe nurses' perceptions of professional dignity, credibility was evaluated. ${ }^{43}$ It was established by accurate purposive sampling of nurses who were working in the environments considered. Dependability was assured by the stability of data collected from October 2012 to March 2014. Conformability was accomplished among different researchers through data accuracy and transferability of the data at broader levels was assured by the data collected in several Italian regions. Reflections and decision-making became apparent via investigative team discussions (face-to-face and Skype), to increase conformability of the interpreted data. A member of the research group who had neither participated in the FGs nor in prior analysis read the original un-coded transcripts of the FGs to validate the results. 


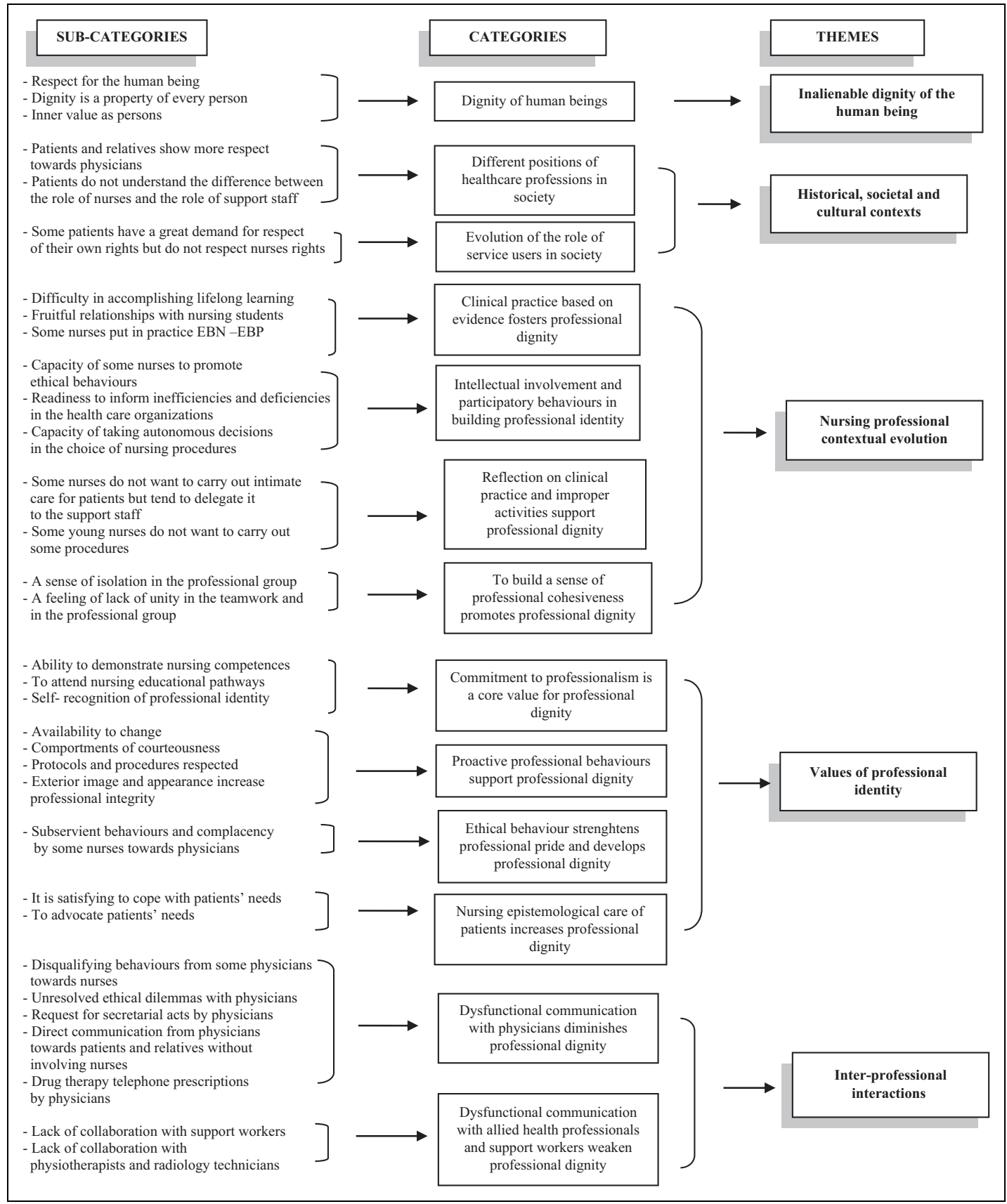

Figure I. Elements that influence the nursing's professional dignity: summary of the analysis. 


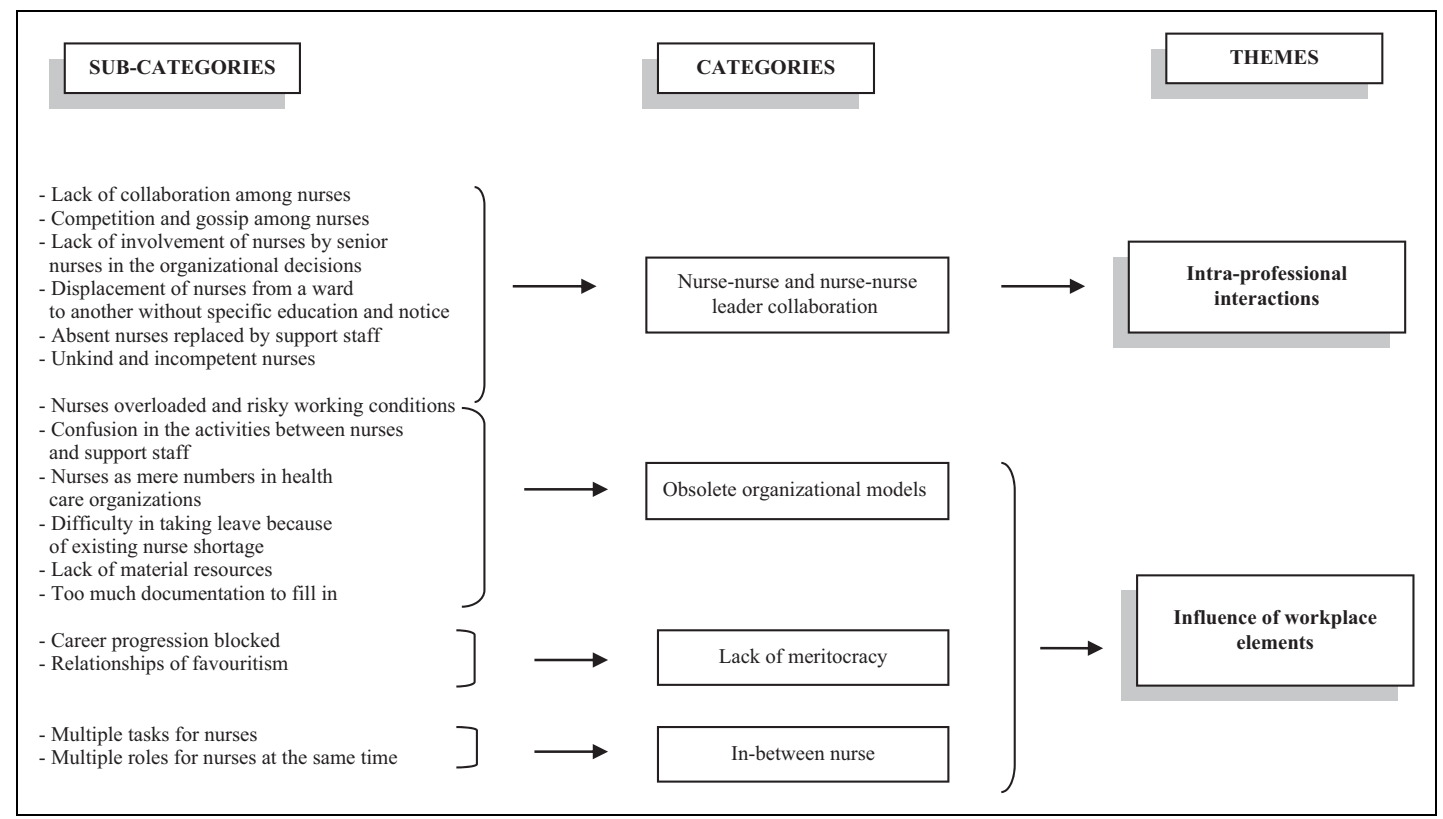

Figure I. continued.

\section{Ethics}

Ethical approval was obtained from the management and review committees of the healthcare facilities used. Before the study, participants were provided with an information sheet and detailed explanation of the study and advised that they were free to refuse to participate or withdraw at any time. Consent forms were signed. Nurses who participated in the study were invited to use a fictitious name; participants were invited to respect professional confidentiality regarding the working experiences that other colleagues would share with them. All the details relating to the participants of the study were kept in locked cabinets and all information remained anonymous.

\section{Findings}

Once the data were analysed and categorized, the perceptions of nurses working in medicine and surgery departments were grouped into seven dimensions concerning nursing's professional dignity. The first four dimensions were as follows:

(a) The inalienable dignity of human beings;

(b) Historical, societal and cultural contexts;

(c) Nursing professional contextual evolution;

(d) Values of professional identity.

The remaining three aspects were related to professional interactions and the influence of workplace factors and were represented by the following:

(e) Inter-professional interactions;

(f) Intra-professional interactions;

(g) Influence of workplace elements. 
Each of these dimensions is now discussed.

(a) The inalienable dignity of human beings

Professional dignity is closely connected with basic human dignity, but develops over time, while a human being's dignity is intrinsic to and inherent in persons:

The dignity, that is, the respect for the person, is basic to all individuals. Before we are professionals we are people and for me it is fundamental to respect the dignity of everyone because we are human beings, living beings. (FG 4)

It is the respect that patients, physicians, everybody who surrounds us, shows us. First of all we have to be respected as persons, and then in our job. (FG 18)

\section{(b) Historical, societal and cultural contexts}

Nurses who participated in the FGs often complained about a lack of social appreciation, and generally about the consideration of the work role by stakeholders:

I was bringing medication to a room just as several relatives were coming out of it. A woman said to a man 'Ah! I would rather be a prostitute than a nurse!'. When I think of professional dignity, I think that the general public and patients too, and sometimes also other professionals, do not realize that the nurses' profession is ... to manage the care plan. For me, the general public does not know what we do. (FG 8)

The mass media were seen as means to highlight only malpractice:

[Italy] is a country where so many clichés are used and the media do not help us and it often happens that when patients are hospitalized ... they only have in their minds what they hear on the news ... That's why we must demonstrate and make an extra effort to make them [patients] understand what professional care is. (FG 4)

\section{(c) Nursing professional contextual evolution}

Changes in nursing legislative and professional development in Italy are in progress since 1991, and this was highlighted by the interviewees:

I think that dignity is related to the knowledge that you have, which has grown over time ... There have been changes over time related to the laws ... and these legislative norms sought to broaden the professional dignity. (FG 6)

(Clinical nurse speaking) The abolition of our job list [Mansionario] has given an impetus to our profession ... I'm more autonomous. Before the doctor arrives, I have done everything, that is, I have done many things which I was not allowed to do before. (FG 15)

\section{(d) The values of professional identity}

The participants experienced a feeling of recognition and respect for their dignity when they worked using their professional knowledge acquired during their basic and post-qualifying training and education:

The dignity of nursing is to be able to care, providing assistance ... to patients using nursing skills and knowledge, which is gained with post-basic training. (FG 17) 
Patient-focused activities appeared to be supportive of raising the dignity of the nursing profession, especially if the activities were carried out in collaboration with other members of the team and with the patient:

When patients go away happy, I am glad that they are satisfied with the care received, that nurses have never treated them as a number or an object. I think this raises the dignity of the profession. The simple fact of calling patients by name and not by the number of the bed ... the patient goes away happy. I think that this has a high value and is important in influencing the dignity of the workers. (FG 7)

The importance of the professional image projected was identified by nurses as essential to achieve greater respect:

Long, very eccentric earrings, nails painted in absurd colors, one can be a very good nurse ... but in the eyes of the patient ... he or she says 'No, no, no! No, I do not want [her] to care for my mother. What? The one with the huge earrings? ... But ... if the nurse is dressed in a simple way, wearing a clean uniform, the shirt tidy, clean shoes, well, not stained with blood ... That gives the professional role a different meaning. (FG 15)

Elements of nursing's professional dignity connected with professional interactions and the influence of workplace aspects were described as follows:

\section{(e) Inter-professional interactions}

In most cases, nurses reported a series of unpleasant events in collaboration with physicians, which greatly abused their professional dignity:

I have my head physician arriving on the ward. Perhaps he calls me by my name, but this has happened only after 20 years because, until recently, he did not even know who I was. He does not know the nurses he works with in the department. He comes, he does not greet anyone, no 'good morning' or 'good evening' or 'how is it going?' Nothing. (FG 11)

Nurses often perceived some physicians dismissing nursing education and post-qualifying courses:

Physicians are offended in their own dignity because we are growing professionally, and very often it bothers them ... Especially if they know that you have passed some specialization courses, or that you are about to go on another course of study. They say 'Where does this nurse want to go; who is he or she?' 'Why do you want to do with this?' 'What is the purpose of this degree?' ... and so they diminish nurses and nursing. (FG 17)

At times, physicians tended to impose their decisions without discussion:

When a physician is telling you 'No, you must do as I say, I'm the consultant' ... I know I am a nurse who is aware of her rights, but it is not so easy [to defend them] [repeated]. (FG 14)

Even working with the support staff was quite confrontational and sometimes offended the dignity of nurses:

Our support worker is always tired. Even after he has done only two or three tasks he has to rest. Whenever one asks something of him, he seems resentful: 'Do I have to do all this? I have to do everything!' . . 'He is not even able to take out an IV line!' ... [The support worker answers usually] 'I cannot take out IV lines; I do not know how to change an adult diaper [... ] I don't know all these things'. (FG 20) 
Our support workers invade the field of competence of nurses without asking. As an example, even for changing large wound dressings they [support workers] decide autonomously, without asking anyone. They [support workers] always deal with the dressings without calling the nurses.

\section{(f) Intra-professional interactions}

Some nurses expressed fixed comportments and refused changes linked to evidence-based practice:

The first sentence you hear from everyone [nurses] telling you when you arrive at a new department, is: 'Here we have always done it like this and we will continue to do it in this way'. That is the most hateful sentence and I do not accept it. We do not have to believe these things. We want to be recognized as nurses in our role; let's stop saying such things. (FG 3)

Unethical behaviours by some nurses were counterproductive in raising professional dignity:

Nurses who are yelling in the hallway, laughing loudly, talking on their cell phones, talking about their business while caring for someone ... there are thousands of examples, as many as you like! Or [another example] 'Well, a patient is a bit confused, he gets treated badly sometimes, even some bad words are used, because maybe this patient can be aggressive. He is not allowed alcohol and ... you get closer and maybe he is aggressive, or he abuses you [verbally], and then you feel entitled to insult him in turn. (FG 1)

The horizontal and vertical abuses described by nurses usually took place among nurses themselves and above all with senior nurses:

Nobody gives a damn if we are happy or not. No one! Support workers, my head nurse, not even my nurse manager. I am a worker with a number that identifies me in this healthcare organization, working 36 hours a week, three shifts, indefinite shifts. They do not even ask if I can. This is too much! (FG 5)

\section{(g) Influence of workplace elements}

Some characteristics connected with the workplace were considered offensive to professional dignity: the constant work overload, the excessive demand for endless documents to fill in and the chronic shortage of staff and material resources:

The work situation can become really dangerous, that is, our work is dangerous. I think about the nurse-to-patient ratio, for the type of patients we have. There is huge external pressure from medical staff, relatives and other nurses who can see what is going on. (FG 2)

In the ward we are two nurses for every shift to care for 28 surgical patients. I would ask any person how we can adhere to care protocols and guidelines even to greet every patient. How can we change this? ... I do not have the time to stop and listen to each patient. (FG 19)

\section{Discussion}

This study provided insights into understanding nursing's professional dignity in internal medicine and surgery departments and enabled basic beliefs about it to be described. The construct examined was deeply embedded in the ontological concept of being a person, connected with the very essence of humanity, which is where the basis of dignity for every individual resides (Figure 2).

Concerning social dignity, ${ }^{11}$ great importance was given to the values that define nursing's professional identity and to the socio-historical background and hence the evolution of nursing in the geographic area 


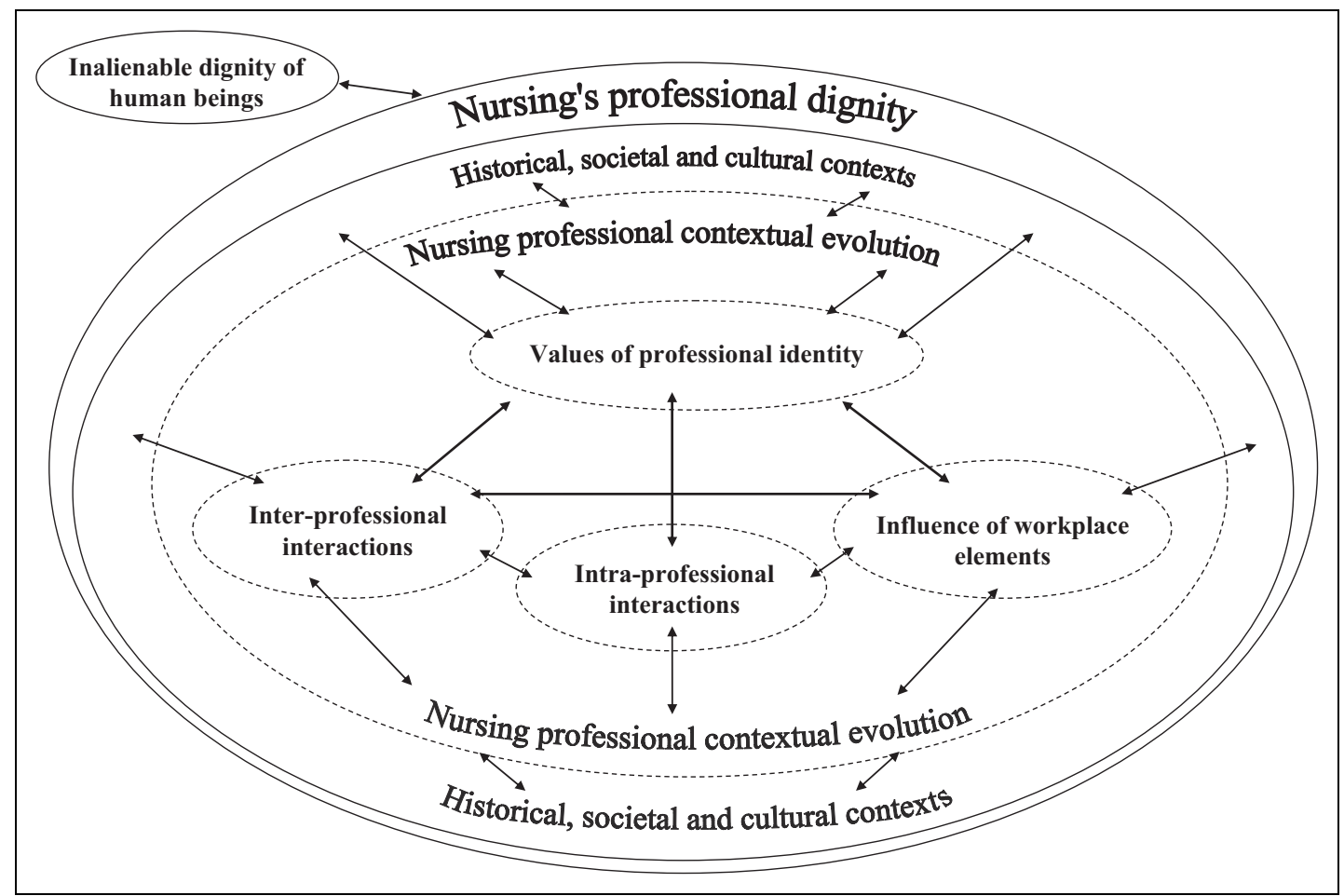

Figure 2. Nursing's professional dignity in internal medicine and surgery departments in hospital settings.

under investigation. The social part of dignity was linked to working with physicians and acknowledged as a key variable. The same could be said of healthcare assistants, who were thought to have a central role in easing the job strain and the burdens of patient care.

Equally important, though, was the relationship with peers and, above all, with senior nurses. Last but not the least, regarding the factors influencing nursing's professional dignity, workplace elements were relevant as background on close examination. The main factors that constitute nursing's professional dignity are as follows:

\section{The inalienable dignity of human beings}

This part of dignity is the ontological aspect linked to the humanity of every person. Nurses as persons possess embedded dignity, and they regard the intrinsic dignity of others as a core value for nursing practice. Human dignity is an inherent value that belongs to every human being as such. The findings showed that social dignity is grounded in human dignity, which goes beyond any social role and represents the intrinsic value of each person in its singularity and specificity.

\section{Historical, societal and cultural contexts}

This contextual part of dignity ${ }^{11}$ forms the common background of the constantly changeable and external elements of dignity. The historical, social and cultural positions of nursing and medicine, expressed in their local healthcare contexts, create the circumstances and experiences for the social part of dignity. This research indicates that internal medicine and surgery departments are often characterized by rigid 
disciplinary boundaries, ${ }^{44}$ maintain historical hierarchies, hinder collaborative teamwork and hamper the development of positive social dignity. These events and circumstances are therefore major features in the study of what constitutes nursing's professional dignity.

\section{Evolution of the nursing professional context}

Nursing is now considered to be an academic profession in Italy, developed in stages and based on legislation. This fact is highlighted by the strongly perceived need by the nurses interviewed to be more respected and recognized in their own role and functions.

The evolution of nursing in Italy happened quickly, is still happening and has had destabilizing effects on nurses. In a relatively short period of time, many normative changes took place, but in practice, especially for hospital nurses, not many things have changed. ${ }^{28}$ The conflicts and struggles experienced by nurses interviewed stressed that the old order ${ }^{45}$ in the professions of the Italian health system is difficult to dismantle.

\section{The values of professional identity}

Intrinsic values held by nurses - such as honesty, integrity, altruism, accountability, moral integrity as professionals, professional comportments, competence, capacities and continuous professional development - were underlined as key tenets to develop professional dignity and professional identity. ${ }^{46,47}$ However, nursing's professional dignity was also seen to be connected with extrinsic values and material aspects of work, such as remuneration, job security, career progression and praise. ${ }^{48}$ Nurses in our study complained about extrinsic values of inadequate income for work done, lack of security at work for themselves and for patients, low quality of care given because of poor nurse-to-patient ratios, difficult work-life balance and irregular hours and shift working. ${ }^{49,50}$

The balance between intrinsic and extrinsic work values is of crucial importance because it affects job satisfaction, recruitment and retention issues. In this light, it is important to recognize that intrinsic values are of the utmost significance for nurses to attain moral integrity as professionals. In our study, participants indicated that intrinsic values are mainly evident in professional autonomy, competence and accountability. However, the extrinsic values, especially better income, were also aspects to be considered urgently. Aspects of nursing's professional dignity connected with professional interactions and the influence of workplace elements are characterized by the following:

\section{Inter-professional interactions}

The long-standing imbalance between nurses and physicians is addressed in numerous researches. ${ }^{51,52}$ The status of medicine and the power and authority possessed by medical staff remains an important feature of healthcare organizations in many countries. ${ }^{53}$ In Europe, studies that emphasized the importance of having an adequate staff-to-patient ratio and a high level of education for nurses to reduce patient mortality were only carried out during the present decade. ${ }^{54-57}$

Despite the fact that collegial relationships within the healthcare teams, healthy work environments ${ }^{58}$ and well-being at work ${ }^{59}$ have been advocated as milestones for effective care, real collaboration between physicians and nurses in the settings scrutinized was an illusion.

Historically, tertiary care in Italy is carried out in situations where medical dominance is characterized by rigid and formal relationships with very poor or non-existent teamwork. In our study, nurses' professional dignity was often severely damaged when nurses were treated with contempt or indifference, or were 
objectified as mere numbers. Relationships with other professions, apart from a few cases, were based on respect. Support workers were considered to be pivotal in easing the job overload.

\section{Intra-professional interactions}

Many studies have described destructive activities in acute care hospitals regarding intra-professional relationships: for example, horizontal violence, verbal abuse, aggression and lack of shared work. ${ }^{60,61}$ It is fundamental to create shared collaborative relationships with colleagues, senior nurses and nurse directors to improve dignity at work. ${ }^{62}$

Nurse-nurse relationships were described in different ways, but above all, there seemed to be little collaboration in the nursing hierarchy. Head nurses and nurse managers were often accused of a lack of responsiveness towards nurses and were often not willing to listen to and recognize nurses' concerns. Although nurse leaders had to be responsive to frontline nurses and to the organizational facility where they worked, ${ }^{63}$ they had minimal decision power compared to other professionals of similar rank. They were often perceived as not engaged enough in initiating change in work environments that are difficult for clinical nurses.

\section{Influence of workplace elements}

Old hierarchical boundaries based on the biomedical model, ${ }^{45}$ unfriendly workplaces, heavy workloads, excessive paperwork, lack of support and other organizational features overlooked or brushed aside nurses' competences and diminished the professional dignity of nurses. The organizational culture of the settings examined was mainly characterized by insufficient opportunities to have real interdisciplinary work ${ }^{64}$ or better transdisciplinary work ${ }^{65}$ and nurses often perceived that their autonomy and decision-making processes were restrained by rigid barriers with other professions, especially medicine.

\section{Comparison of nursing's professional dignity and its expression in internal medicine and surgery departments with a previously developed model}

The results from this research were compared with a previous study about nursing's professional dignity. ${ }^{18}$ The model presented in the earlier study was divided into two macro-dimensions. The first macrodimension was based on the characteristics of human beings. The second macro-dimension referred to the workplace elements that fostered nursing's professional dignity. This study uncovered a clearly missing element in the first macro-dimension, that is, the personal dignity embedded in the concept of self, ${ }^{66,67}$ for example, self-respect and self-esteem did not emerge as factors for the construction of the nurses' perceptions of professional dignity in internal medicine and surgery departments. The new and important elements that came to light or had a stronger emphasis were mainly related to the second aspect of the earlier framework $^{18}$ and were as follows:

- The importance laid on the historical, societal and cultural backgrounds that shape the evolution of different professions in a society. ${ }^{44}$ In this regard, the position of nursing in its cultural development in Italy was seen as central to understand the topic under analysis.

- The interactions between nurses and physicians and between clinical nurses and nurses in senior positions. ${ }^{68,69}$ These elements, well-known key factors to attain mutual respect, communication and collaboration among professionals, were significant to understanding professional dignity and its respect for nurses in the settings examined. ${ }^{70}$

- The collaboration with healthcare assistants was also recognized as an important component that could help nurses to focus on their scope of practice. ${ }^{71}$ Support workers were seen as essential to 
lighten the work strain and overload. Unfortunately, the lack of collaboration resulted in hindering the development of nursing in the present scenario.

- The organizational elements of the workplace, ${ }^{72}$ such as the strain of shiftwork, work overload and poor nurse ratios, were thought to be pivotal in improving professional dignity for nurses.

\section{Conclusion and recommendations}

The agenda to realize a significant transformation in the delivery of healthcare is being motivated by the need to provide quality, affordable and sustainable healthcare. Healthcare facilities have the mission to improve inter- and intra-professional interactions in order to ameliorate the ethical climate and have better health outcomes for patients with useful cost-containment. Despite this, the collaboration among professionals is still an ongoing issue in the Italian hospital system where many environments are still stuck in an old regime.

The organizational environments included in our study, with their low staffing levels, time constraints, job exertion and overburden, did not promote respect for the dignity of the nursing profession. A defensive culture, even if aspects of innovation are visible, ${ }^{73,74}$ is still strong and measures to advance speedier changes of the situation should be put into practice. One of these could be to improve inter-professional education, ${ }^{75,76}$ which is as yet completely lacking in the Italian educational system; or to have the moral courage ${ }^{77}$ to report, at least, the worst improper behaviours of healthcare professionals.

Future developments of this research should be to explore the various elements of nursing's professional dignity in depth and in other healthcare settings. Professional dignity for nurses has been shown to be a complex concept influenced by contextual elements of the society in which this construct is developed. To have a deeper comprehension of it would be beneficial to obtain mutual respectful interactions among professionals. $^{78,79}$

\section{Limitations}

Several biases could be detected in the preparation, organization and reporting phases of this research. ${ }^{80}$ In the preparation phase, in the sampling strategy, the purposive selection method failed to include one or two study outliers (physicians, physiotherapists, etc.), who could have had markedly different opinions about the argument under study. These health professionals would have been an important source for understanding different points of view on the main elements factoring nursing's professional dignity.

In the organization stage, although emerging themes were discussed at length by the research group, some categories tended to overlap, due to the strong interconnectedness among concepts, especially regarding the workplace elements.

In the reporting phase, not all similarities and differences among themes originating in the earlier theoretical model ${ }^{18}$ were systematically described and reported respect to this new empirical research because of the intrinsic complexities to compare aspects of the topic under study.

\section{Conflict of interest}

No conflict of interest.

\section{Funding}

This research received no specific grant from any funding agency in the public, commercial, or not-for-profit sectors. 


\section{References}

1. Kolnai A. Dignity. Philosophy 1976; 51: 251-271.

2. Sensen O. Human dignity in historical perspective: the contemporary and traditional paradigms. Eur J Polit Theor 2011; 10(1): 71-91.

3. Misztal BA. The idea of dignity: its modern significance. Eur J Soc Theor 2013; 16(1): 101-121.

4. Ashcroft RE. Making sense of dignity. J Med Ethics 2005; 31: 679-682.

5. Franklin LL, Ternestedt BM and Nordenfelt L. Views on dignity of elderly nursing home residents. Nurs Ethics 2006; 13(2): 130-146.

6. Andorno R. Human dignity and human rights as a common ground for a global bioethics. J Med Philos 2009; 34(3): 223-240.

7. Nordenfelt L. The varieties of dignity. Health Care Anal 2004; 12(2): 69-89.

8. Nordenfelt L and Edgar A. Dignity and older Europeans: The four notions of dignity. Qual Ageing: Policy Pract Res 2005; 6(1): 17-21.

9. Smith AM. Human dignity and the common good in the aristotelian-thomistic tradition. New York: The Edwin Mellen Press, 1995.

10. Pelaéz M. L'arte di vivere bene: beni, virtù, norme [The art of good living: good, virtues, rules]. Milano: Ares, 2007 (in Italian).

11. Jacobson N. Dignity and health: a review. Soc Sci Med 2007; 64: 292-302.

12. Sayer A. Dignity at work: broadening the agenda. Organization 2007; 14(4): 565-581.

13. Edlund M, Lindwall L, von Post I, et al. Concept determination of human dignity. Nurs Ethics 2013; 20(8): 851-860.

14. Lindwall $\mathrm{L}$ and von Post I. Preserved and violated dignity in surgical practice - nurses' experiences. Nurs Ethics 2014; 21(3): 335-346.

15. Vanlaere L and Gastmans CA. A personalist approach to care ethics. Nurs Ethics 2011; 18: 161-173.

16. Laschinger HKS, Finegan J, Shamian J, et al. Impact of structural and psychological empowerment on job strain in nursing work settings: expanding Kanter's model. J Nurs Adm 2001; 31: 260-272.

17. Mordacci R. Rispetto [Respect]. Milano: Raffaello Cortina Editore, 2012 (in Italian).

18. Sabatino L, Stievano A, Rocco G, et al. The dignity of the nursing profession: a meta-synthesis of qualitative research. Nurs Ethics 2014; 21: 659-672.

19. Jacobson N. A taxonomy of dignity: a grounded theory study. BMC Int Health Hum Right 2009; 9: 3.

20. Lin YP, Tsai YF and Chen HF. Dignity in care in the hospital setting from patients' perspectives in Taiwan: a descriptive qualitative study. $J$ Clin Nurs 2011; 20: 794-801.

21. Lin YP, Watson R and Tsai YF. Dignity in care in the clinical setting: a narrative review. Nurs Ethics 2012; 20(2): $168-177$.

22. Khademi M, Mohammadi E and Vanaki Z. Nurses' experiences of violation of their dignity. Nurs Ethics 2012; 19 : 328-340.

23. Tschudin V. Nurses matter: reclaiming our professional identity. London: Macmillan Press, 1999.

24. Öhlén J and Segesten K. The professional identity of the nurse: concept analysis and development. J Adv Nurs 1998; 28: 720-727.

25. Haslam SA, Jetten J, Postmes T, et al. Social identity, health and well-being: an emerging agenda for applied psychology. Appl Psychol 2009; 58(1): 1-23.

26. Rolfe G. Postmodernism for healthcare workers in 13 easy steps. Nurse Educ Today 2001; 21: 38-47.

27. Sala R and Manara D. The regulation of autonomy in nursing: the Italian situation. Nurs Ethics 1999; 6(6): 451-467.

28. Rocco G, Affonso D, Mayberry L, et al. Evolution of professional nursing culture in Italy: metaphors and paradoxes. Global Qual Nurs Res 2014; 1. DOI: 10.1177/2333393614549372.

29. Demir D and Rodwell J. Psychosocial antecedents and consequences of workplace aggression for hospital nurses. J Nurs Scholarsh 2012; 44(4): 376-384. 
30. Yildirim D. Bullying among nurses and its effects. Int Nurs Rev 2009; 56: 504-511.

31. Camerino D, Estryn-Béhar M, Conway PM, et al. Work-related factors and violence among nursing staff in the European NEXT study: a longitudinal cohort study. Int J Nurs Stud 2008; 45(1): 35-50.

32. Skei K. Collaboration at risk: registered nurses' experiences on orthopaedic wards. J Clin Nurs 2008; 17: 1907-1914.

33. Miller KL, Reeves S, Zwarenstein M, et al. Nursing emotion work and interprofessional collaboration in general internal medicine wards: a qualitative study. $J$ Adv Nurs 2008; 64: 332-343.

34. Reeves S, Rice K, Gotlib Conn L, et al. Interprofessional interaction, negotiation and neo-negotiations on general internal medicine wards. $J$ Interprof Care 2009; 23(6): 633-645.

35. Leever AM, Hulst MVD, Berendsen AJ, et al. Conflicts and conflict management in the collaboration between nurses and physicians - a qualitative study. $J$ Interprof Care 2010; 24(6): 612-624.

36. Tang CJ, Chan SW, Zhou WT, et al. Collaboration between hospital physicians and nurses: an integrated literature review. Int Nurs Rev 2013; 60: 291-302.

37. Stievano A, De Marinis MG, Russo MT, et al. Professional dignity in nursing in clinical and community workplaces. Nurs Ethics 2012; 19: 341-356.

38. Dierckx de Casterlé B, Gastmans C, Byron E, et al. QUAGOL: a guide for qualitative data analysis. Int J Nurs Stud 2012; 49: 360-371, http://dx.doi.org/10.1016/j.jinurstu.2011.09.012

39. Hsieh H and Shannon SE. Three approaches to qualitative content analysis. Qual Health Res 2005; 15(9): $1277-1288$.

40. Graneheim UH and Lundman B. Qualitative content analysis in nursing research: concepts, procedures and measures to achieve trustworthiness. Nurse Educ Today 2004; 24: 105-112.

41. Morse J. Constructing qualitatively derived theory: concept construction and concept typologies. Qual Health Res 2004; 14: 1387-1395.

42. Lipscomb M. Abductive reasoning and qualitative research. Nurs Philos 2012; 13(4): 244-256.

43. Lincoln YS and Guba EG. Naturalistic inquiry. Newbury Park, CA: Sage, 1985.

44. Hall P. Interprofessional teamwork: professional cultures as barriers. J Interprof Care 2005; 19: 188-196.

45. Svensson R. The interplay between doctors and nurses - a negotiated order perspective. Sociol Health Ill 1996; 18 : 379-398.

46. Horton K, Tschudin V and Forget A. The value of nursing: a literature review. Nurs Ethics 2007; 14(6): 716-740.

47. Hegney D, Plank A and Parker V. Extrinsic and intrinsic work values: their impact on job satisfaction in nursing. J Nurs Manag 2006; 14(4): 271-281.

48. Vansteenkiste M, Neyrinck B, Niemiec CP, et al. On the relations among work value orientations, psychological need satisfaction and job outcomes: a self-determination theory approach. J Occup Organ Psych 2007; 2: 251-277.

49. Caricati L, La Sala R, Marletta G, et al. Work climate, work values and professional commitment as predictors of job satisfaction in nurses. J Nurs Manag 2014; 22: 984-994.

50. Ambrosi E, Portoghese I, Galletta M, et al. Turnover intention among nurses with $\leq 3$ years of work experience: an exploratory study. Assist Inferm Ric 2011; 30: 126-134, http://dx.doi.org/10.1702/970.10586 (in Italian).

51. Price S, Doucet S and McGillis Hall L. The historical social positioning of nursing and medicine: implications for career choice, early socialization and interprofessional collaboration. J Interprof Care 2014; 28(2): 103-109.

52. Reeves S, Nelson S and Zwarenstein M. The doctor-nurse game in the age of interprofessional care: a view from Canada. Nurs Inq 2008; 15(1): 1-2.

53. Hojat M, Gonnella JS, Nasca TJ, et al. Comparisons of American, Israeli, Italian and Mexican physicians and nurses on the total and factor scores of the Jefferson scale of attitudes toward physician-nurse collaborative relationships. Int J Nurs Stud 2003; 40: 427-435.

54. Sermeus W, Aiken LH, Van den Heede K, et al. Nurse forecasting in Europe (RN4CAST): rationale, design, and methodology. BMC Nurs 2011; 10: 6. 
55. Aiken LH, Sermeus W, Van den Heede K, et al. Patient safety, satisfaction, and quality of hospital care: cross-sectional surveys of nurses and patients in 12 countries in Europe and the United States. BMJ 2012; 344: e1717.

56. Aiken LH, Sloane DM, Bruyneel L, et al. Nurses' reports of working conditions and hospital quality of care in 12 countries in Europe. Int J Nurs Stud 2013; 50: 143-153.

57. Aiken LH, Sloane DM, Bruyneel L, et al. Nurse staffing and education and hospital mortality in nine European countries: a retrospective observational study. Lancet 2014; 383: 1824-1830.

58. Twigg $\mathrm{D}$ and McCullough $\mathrm{K}$. Nurse retention: a review of strategies to create and enhance positive practice environments in clinical settings. Int J Nurs Stud 2013, http://dx.doi.org/10.1016/j.ijnurstu.2013.05.015

59. Utriainen K and Kyngäs H. Hospital nurses' job satisfaction: a literature review. J Nurs Manag 2009; 17(8): 1002-1010.

60. Hutchinson M, Vickers M, Jackson D, et al. Workplace bullying in nursing: towards a more critical organizational perspective. Nurs Inq 2006; 13: 118-126.

61. Becher J and Visovsky C. Horizontal violence in nursing. Medsurg Nurs 2012; 4: 201-213.

62. Storch JL and Kenny N. Shared moral work of nurses and physicians. Nurs Ethics 2007; 14(4): 478-491.

63. Schick Makaroff K, Storch J, Pauly B, et al. Searching for ethical leadership in nursing. Nurs Ethics 2014; 21 : 642-658.

64. Reeves S, Rice K, Gotlib Conn L, et al. Interprofessional interaction, negotiation and non-negotiation in general internal medicine wards. J Interprof Care 2009; 23(6): 633-645.

65. Baxter SK and Brumfitt SM. Professional differences in interprofessional working. $J$ Interprof Care 2008; 22 : 239-251.

66. Yalden BJ and McCormack B. Constructions of dignity: a pre-requisite for flourishing in the workplace? Int $J$ Older People Nurs 2010; 5: 137-147.

67. Olthuis G, Leget C and Dekkers W. Why hospice nurses need high self-esteem. Nurs Ethics 2007; 14: 62-71.

68. Matziou V, Vlahioti E, Perdikaris $\mathrm{P}$, et al. Physician and nursing perceptions concerning interprofessional communication and collaboration. $J$ Interprof Care 2014; 28: 526-533.

69. Gormley DK. Are we on the same page? Staff nurse and manager perceptions of work environment, quality of care and anticipated nurse turnover. J Nurs Manag 2011; 19(1): 33-40.

70. Hughes B and Fitzpatrick J. Nurse-physician collaboration in an acute care community hospital. J Interprof Care 2010; 24: 625-632.

71. Rhéaume A. The changing division of labour between nurses and nursing assistants in New Brunswick. $J$ Adv Nurs 2003; 41(5): 435-443.

72. Lavoie-Tremblay M, Wright $\mathrm{D}$, Desforges $\mathrm{N}$, et al. Creating a healthy workplace for new-generation nurses. J Nurs Scholarsh 2008; 40(3): 290-297.

73. France G and Taroni F. The evolution of health-policy making in Italy. J Health Polit Policy Law 2005; 30(1-2): 169-188.

74. Tousijn W. Medical dominance in Italy: a partial decline. Soc Sci Med 2002; 55(5): 733-741.

75. Hall P and Weaver L. Interdisciplinary education and teamwork. A long and winding road. Med Educ 2001; 35 : $867-875$.

76. World Health Organization. Framework for action on interprofessional education and collaborative practice, http:// whqlibdoc.who.int/hq/2010/WHO_HRH_HPN_10.3_eng.pdf (2010, accessed 21 August 2014).

77. Hawkins SF and Morse J. The praxis of courage as a foundation for care. J Nurs Scholarsh 2014; 46: 263-270.

78. Antoniazzi CD. Respect as experienced by registered nurses. West J Nurs Res 2011; 33: 745-766.

79. Bournes DA and Milton CL. Nurses' experiences of feeling respected - not respected. Nurs Sci Quart 2009; 22 : $47-56$.

80. Elo S, Kääriäinen M, Kanste O, et al. Qualitative content analysis: a focus on trustworthiness. Qual Health Res 2014; 24: 1-10. 